\title{
Synthesis and Characterization of Nano-pyramid Decorated ZnO Nanowires
}

\author{
Dinghao Tang*, Lawrence F. Allard** and Jingyue (Jimmy) Liu* \\ * Center for Nanoscience and Department of Physics and Astronomy, University of Missouri-St. \\ Louis, One University Boulevard, St. Louis, Missouri 63121 \\ ** Materials Science and Technology Div., Oak Ridge National Laboratory, Oak Ridge, TN 37831
}

The wide-band gap (3.37 eV) semiconductor $\mathrm{ZnO}$ possesses unique physicochemical properties and has broad applications in electronics, optoelectronics, energy, sensing and catalysis. $\mathrm{ZnO}$ nanostructures such as wires, belts, rings, helixes and other types of structures have been recently synthesized [1]. The rich family of hierarchical $\mathrm{ZnO}$ nano/micro structures not only provides important applications but also provides a system for studying the fundamental nature of nucleation, growth and self-assembly of nanocomponents. Synthesis of surface-architectured nanostructures is critical to developing nanoscale sensors nanocatalysts. We report here our recent progress on synthesizing nano-pyramid decorated $\mathrm{ZnO}$ nanowires.

The $\mathrm{ZnO}$ nanostructures were grown by a standard thermal evaporation-condensation process [2]. To better control the surface morphology and the composition of the synthesized oxide nanostructures we used a mixture of oxygen (0-10\%) and nitrogen or argon as the carrier gas. The morphology of the $\mathrm{ZnO}$ nanostructures was examined in a high-resolution field emission SEM, and their atomic structures were characterized in a JEOL 2200FS TEM/STEM equipped with a CEOS GmbH. aberration corrector on the probe-forming optics that provides a nominal probe diameter for STEM imaging of $0.07 \mathrm{~nm}$.

Figure 1a shows a secondary-electron (SE) image of the synthesized $\mathrm{ZnO}$ nanowires decorated with nanoscale pyramids on its side surfaces. Detailed analysis showed that the diameters of the nanopyramid decorated nanowires range from $0.8 \mu \mathrm{m}$ to $2 \mu \mathrm{m}$ and their lengths range from $40 \mu \mathrm{m}$ to about $0.5 \mathrm{~mm}$. All the nano-pyramid decorated $\mathrm{ZnO}$ nanowires are extremely straight with growth directions along the $\mathrm{ZnO}$ [0001] direction. Figure 1b shows a high-resolution SE image of the nanoscale pyramids, showing that the base plane of the nano-pyramids is the $\mathrm{ZnO}(0001)$ basal pane. Measurements of many nano-pyramids, imaged in plan view, revealed that the angle between the basal plane and the side planes of the nano-pyramids is about $72^{\circ}$, which suggests that the side planes of the nano-pyramids are probably the $\mathrm{ZnO}\{11-21\}$ pyramid planes. Figure 1c shows a SE image of the growth end of a nano-pyramid decorated $\mathrm{ZnO}$ nanowire, clearly revealing the hexagonal shape of the nanowire and its flat basal plane. Figure 2a shows a STEM bright-field image of a truncated nano-pyramid and Figs. $2 b$ and $2 c$ show high-resolution STEM images of the base and side surfaces of the nano-pyramid, respectively. The corresponding optical diffractograms are shown in the insets. Analysis of the diffractograms showed that the base plane of the nanopyramids is the $\mathrm{ZnO}\{0001\}$ plane and the side surfaces are composed of $\{11-21\}$ planes or $\{11-22\}$ planes. A detailed structural model of the observed nano-pyramids and their growth mechanisms will be discussed [3].

\section{References}

[1] Z.L. Wang, J. Phys.: Condens. Matter, 16 (2004) R829.

[2] Z. W. Pan et al., Science 291 (2001) 1947.

[3] DT and JL acknowledge the funding of this research by the University of Missouri-St. Louis; research at Oak Ridge National Laboratory's High Temperature Materials Laboratory was sponsored by the U. S. DOE, Office of Energy Efficiency and Renewable Energy, Vehicle Technologies Program. 

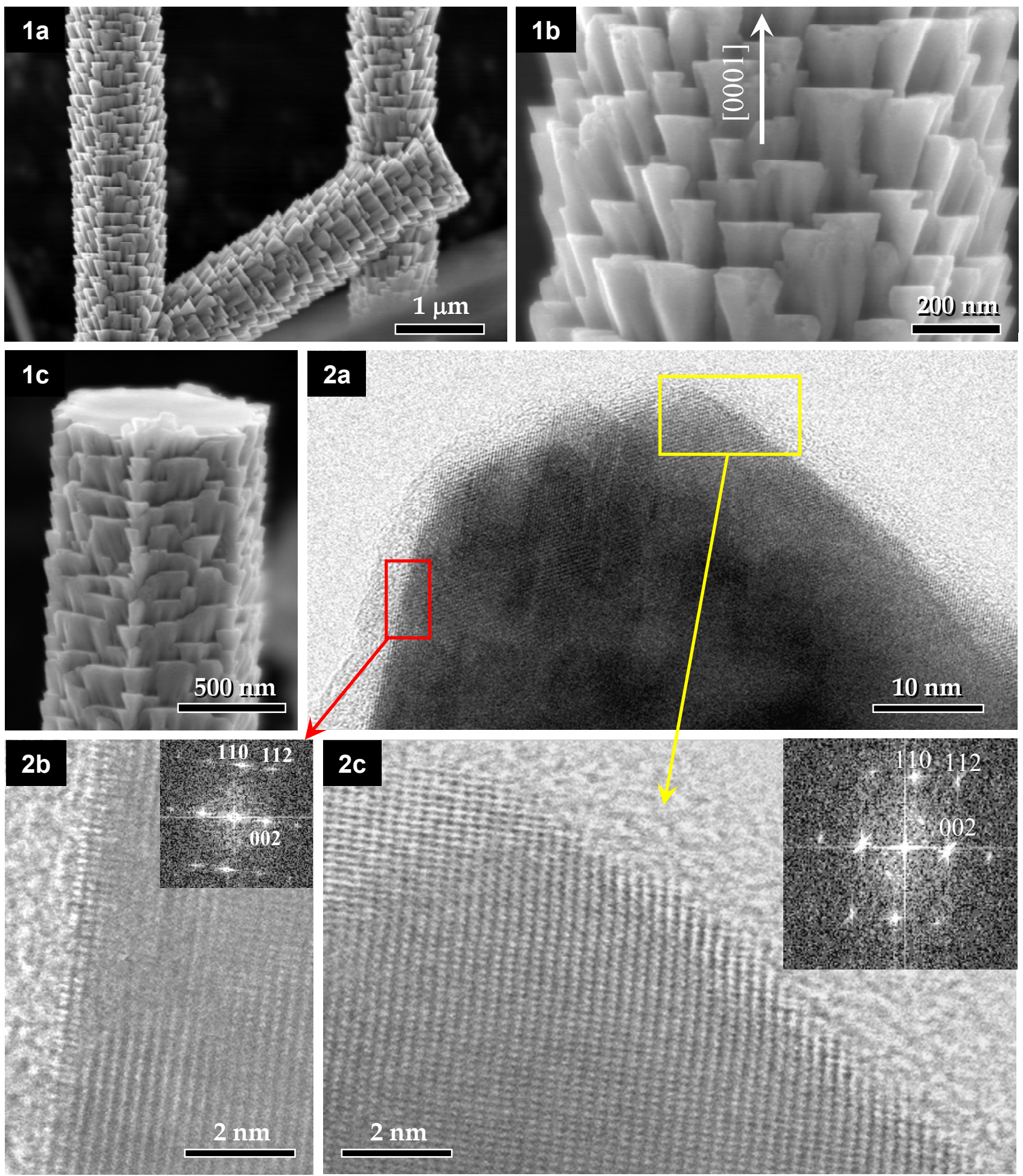

Fig. 1. SEM images of nano-pyramid decorated $\mathrm{ZnO}$ nanowires.

Fig. 2. STEM BF image (a) and high-resolution images (b and c) of a truncated nano-pyramid show the atomic arrangement of the base and side surfaces of the nano-pyramids. The corresponding optical diffractograms are shown in the insets of images (b) and (c), respectively. The growth direction of the nanowire and the nano-pyramid is along the $\mathrm{ZnO}$ [001] direction. 